\title{
Delft Testbed Interferometer: a homothetic mapping test setup
}

\author{
Hedser van Brug, Bastiaan Oostdijck, Teun van den Dool, Peter Giesen, and Wim Gielesen
}

TNO TPD, Stieltjesweg 1, 2628 CK Delft, the Netherlands

\begin{abstract}
The Delft Testbed Interferometer (DTI) will be presented. The basics of homothetic mapping will be explained together with the method of fulfilling the requirements as chosen in the DTI setup. The optical layout incorporates a novel tracking concept enabling the use of homothetic mapping in real telescope systems for observations on the sky. The requirements for homothetic mapping and the choices made in the DTI setup will be discussed. Finally the planned experiments will be discussed.
\end{abstract}

Keywords: Multi aperture interferometry, wide field imaging, homothetic mapping

\section{INTRODUCTION}

At TPD (the institute of applied physics) of TNO (a Dutch organization founded as an interface between the scientific research at the Dutch universities and the knowledge demands of the Dutch industries), there is a research effort going on indicated as the Knowledge center for Aperture Synthesis (KAS).

KAS is a joined effort of TNO TPD with Space Research Organization Netherlands (SRON), Observatory Leiden, Delft University of Technology, European Space Agency (ESA), and European Southern Observatory (ESO). Presently running research includes investigations in the field of delay lines, achromatic nulling, adaptive optic systems, and optical aperture synthesis.

The research in the field of optical aperture synthesis focuses at homothetic mapping. To investigate the possibilities and the difficulties associated with homothetic mapping it was decided to construct an optical test bed that will demonstrate that homothetic mapping is possible. Once this has been proven the test bed will be available as guest facility for e.g. graduate and $\mathrm{PhD}$ students.

This paper will first provide an introduction to the concept of homothetic mapping, followed by a detailed description of the DTI system. All choices will be explained and the restrictions that we have adopted will be discussed. At the end the planned experiments will be presented.

\section{HOMOTHETIC MAPPING}

Homothetic mapping is one of the types of combining beams from separate telescopes. In homothetic mapping the beams entering the combination system form a scaled down version of the beams as captured by the telescopes. This means that the layout of the telescopes is copied to the combination stage, after scaling, and that the beam diameters are scaled by the same factor. The beams are then combined as if they are coming from one large telescope that has an aperture plate in a pupil plane with the same number of apertures in it as there are actual telescopes.

Beams that are originating from the same star but enter via different telescopes have to be mutually parallel upon entering the combination system. Beams from a different star should also be parallel to each other but will have an angular difference with all beams from other stars. In homothetic mapping each star is imaged and, since the system functions as if a single capturing telescope with an aperture plate was used, all images will show fringes. In this way the combined telescopes can be used in wide field imaging.

Further author information:

Hedser van Brug: E-mail: brug@tpd.tno.nl, Telephone: +31 152692489 
Important issues for a homothetic beam combination are: beam overlap, pointing, OPD, pupil rotation, and pupil magnification.

Pupil configuration, mapping This is the requirement on the actual layout of the beams during recombination. The beams should be arranged as the telescopes that were used to capture the beams.

Beam overlap In a general homothetic mapping system the entrance pupils will be imaged onto the exit pupils where the pupils will be scaled down in size. The beams pertaining to different stars should overlap in the exit pupil plane.

Pointing The beams, pertaining to the same star, propagating via different optical paths should have equal pointing direction when entering the combination system.

OPD Since fringes have to be measured, and since 'white light' is used, the separate optical path lengths should be very accurately equal.

Pupil rotation The beams should not be rotated about the propagation direction, other than a common rotation. The common rotation has to be known accurately since the pupil configuration will have to be rotated over the same angle. A rotation of one of the beams leads to poorly overlapping star images.

Pupil magnification As holds for the pupil rotation, also the pupil magnification has to be known accurately and has to be equal for all beams. If one beam experiences a different magnification than the other beams this will lead to pointing errors and therefore poor star image overlap.

\section{OPTICAL LAYOUT}

In the Delft Testbed Interferometer three telescopes are simulated where the beams are chosen to have a diameter of $10 \mathrm{~mm}$ and a baseline of about $120 \mathrm{~mm}$. In this way the size and baseline are a scaled copy of the VLT-I system in Chili. The field of view is chosen to be at maximum 800 arcsec, scaled with respect to the VLT-I by the same factor as the beam size and baseline. Note that a reduction in beam and baseline size demands an increase by the same amount in field of view.

Since DTI is a breadboard setup we have to create our own stars. This task is accomplished via fibres acting as point sources placed in the focal plane of a parabolic mirror (focal length $600 \mathrm{~mm}$ ). Each out-coupled beam from a fibre leads to a collimated beam with its own propagation direction. The placement of the fibre ends determines the field of view.

As light sources we will use a HeNe laser during initial alignment, an SLD (super luminescent diode) during the preliminary mapping experiments, and a white light (Xe) source for the actual experiments.

From the collimated beams, diameter about $150 \mathrm{~mm}$, three sub beams (diameter $10 \mathrm{~mm}$ ) are selected via an aperture plate. The three sub beams are guided via three equal optical paths, consisting of a delay line and two correction mirrors, towards the mapping stage. This mapping stage consists in case of the DTI of three mirrors that are configured in a mirrored copy of the apertures. The whole DTI is shown in Fig.1. The shown figure is an output version of the mechanical design package.

In order to explain the working principle of the DTI system, it is best to give each part of the setup a descriptive name. The fiber holder combined with the off-axis paraboloid will be referred to as the Star Simulator (SS). The large flat mirror that directs the light coming from the SS downwards, towards the optical table, is the Star Scanning Mirror (SSM). The three small mirrors below the SSM are the Aperture Mirrors $(\mathrm{AM})$. These mirrors direct the light, passing through the three apertures in the aperture plate towards the Delay Lines (DL). From the DLs the light propagates over the Correction Mirrors (CM), six mirrors used as periscope to define the place on the Mapping Mirrors (MM), two mirrors for each of the three beams. The MM are aligned equal to the (front to back inversion) AMs. Finally, the light that is reflected upwards by the MMs is reflected by the Matching Scanning Mirror (MSM) towards the Imaging Telescope (IT), consisting of an off-axis paraboloid mirror and a hyperboloid. Together, the paraboloid and hyperboloid form a 5 meter telescope. The IT images the light on a CCD camera (digital, $1024 \times 1024$ pixels). 


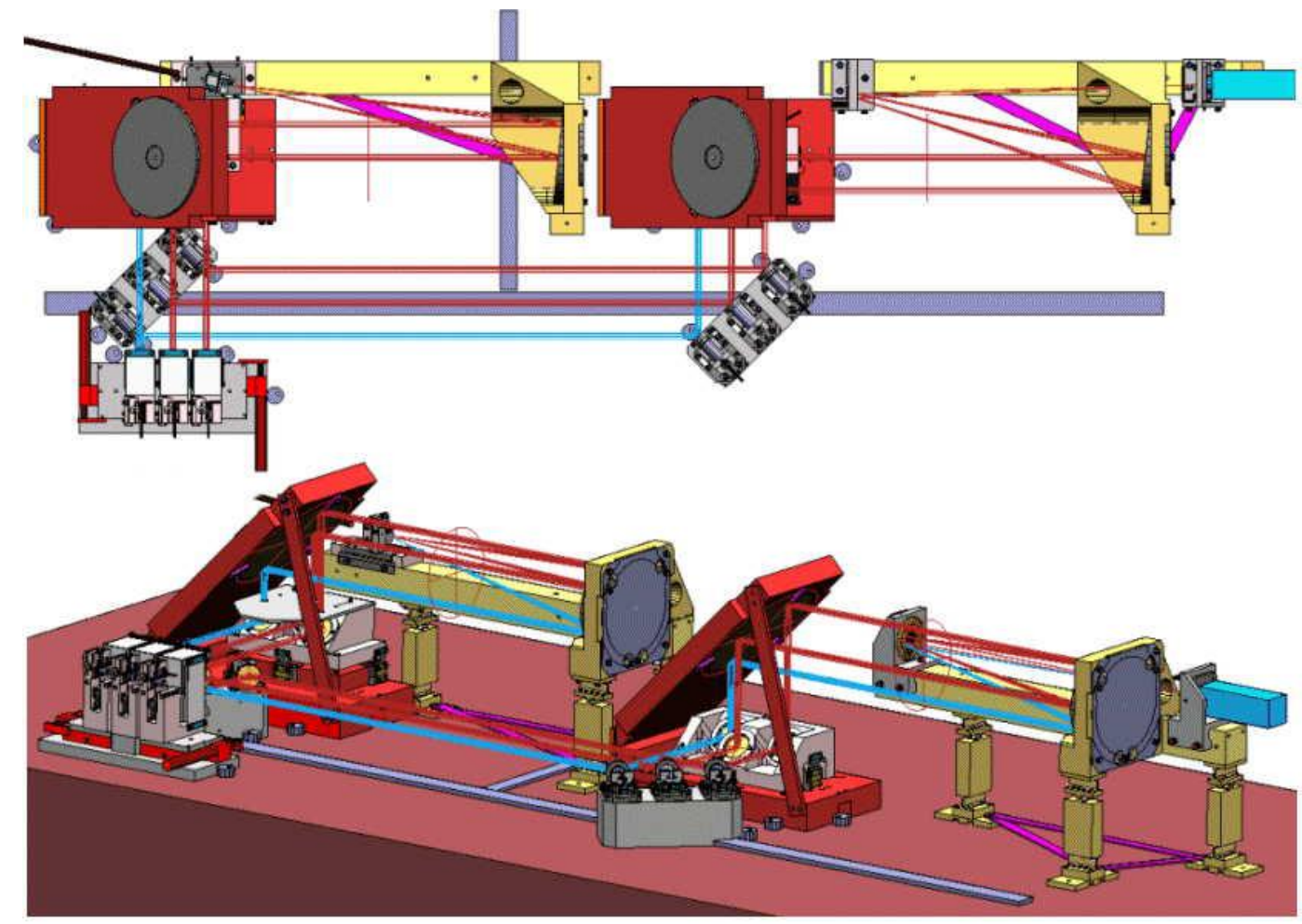

Figure 1. Artist's impression of the DTI system, as coming from the mechanical design program. At the top a top-view is shown, and at the bottom a 3D-view. The footprint of the system is about $220 \times 75 \mathrm{~cm}$, and the height equals $43 \mathrm{~cm}$

As can be seen from Fig.1 the Aperture Mirrors beneath the aperture plate (left hand side of the layout), are mirrored in terms of layout at the mapping stage (right hand side, Mapping Mirrors). The MMs are positioned beneath the large mirror on the right hand side. The mirroring operation is required since the chosen layout flips the field.

As stated above, between the AM and the MM there are Delay Lines and two Correction Mirrors per beam path. For the DLs we have chosen for the simple roof-top approach, see Fig.2. The DLs are used for the course Optical Path Difference (OPD) correction, to redirect the light at a lower height, and importantly to correct for pupil rotations. The OPD accuracy of the DLs will be in the order of a micrometer. The Correction Mirrors have three tasks. The first is to position the beams accurately on the Mapping Mirrors. The alignment accuracy in position is about $3 \mu \mathrm{m}$. The second task is to give the three sub beams equal directions, i.e. to reduce the pointing error to a value smaller than $5 \mu \mathrm{rad}$. The third task is to correct for OPD errors such that the optical paths are equal to within $20 \mathrm{~nm}$. For these tasks the CM are placed on piezo actuators that allow tip/tilt and piston corrections. The OPD correction will also lead to small mapping errors but these induced errors are several orders of magnitude smaller than the allowed range for mapping errors. The stated required accuracies follow from the error budget calculations (see Table 1) to arrive at a phase retrieval goal of $\lambda / 100$. The phase will be retrieved from the fringes in the images of the stars.

The error budget has been presented previously and for a full discussion on the error budget the reader is referred to that paper. ${ }^{1}$ Table 1 gives for all found error sources the allowed error, and the effect they will then have on the accuracy $(\Delta \varphi)$ with which the phase can be measured. From this error budget it follows that the phase can be measured with an accuracy of better than $\lambda / 100$.

During the use of the DTI system we will use an active OPD control by actuating the piezo's on which the correction mirrors are glued. In total we have six CMs and only four of them are actuated via piezo's. The 


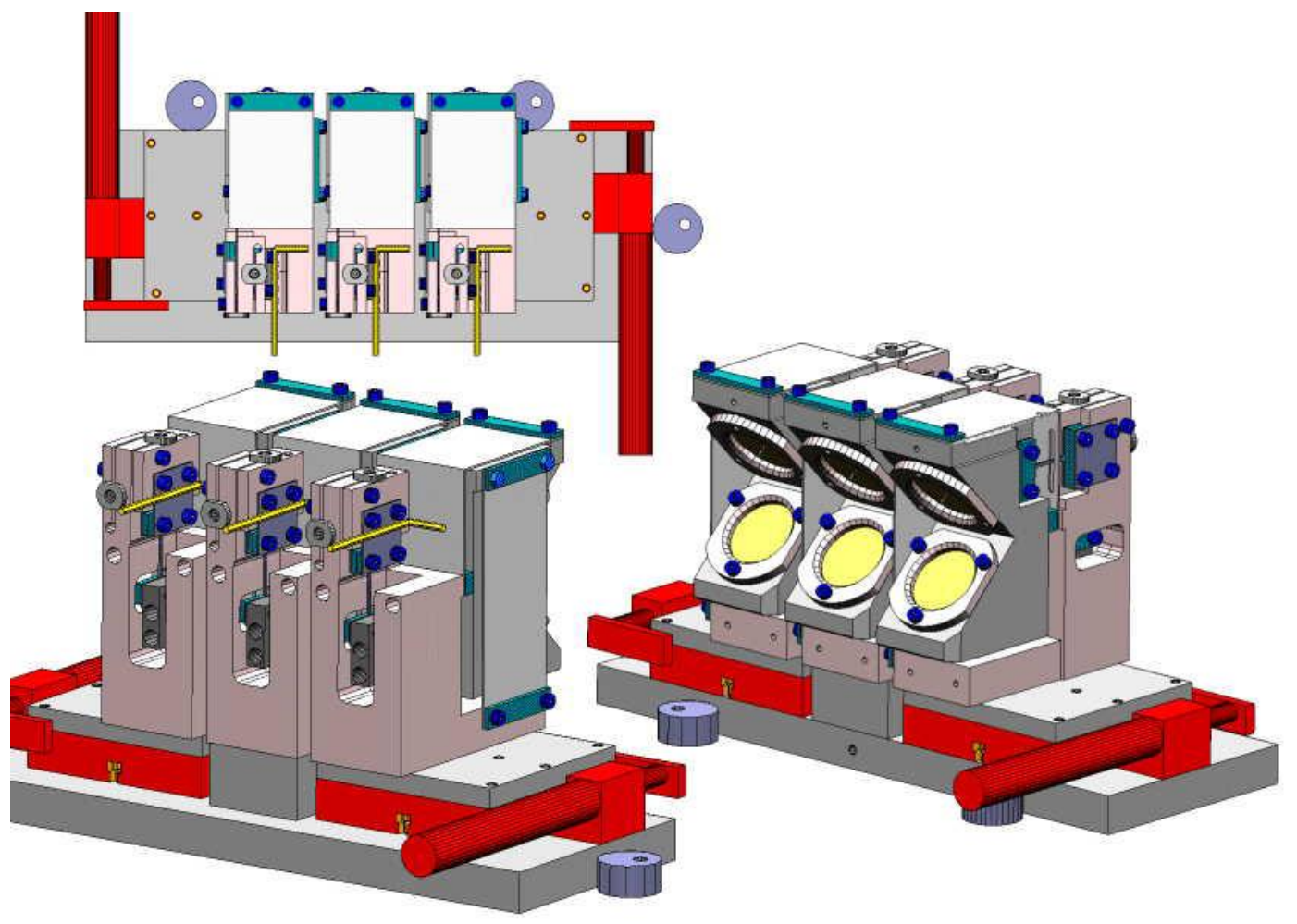

Figure 2. Impression of the roof-top type delay lines

Table 1. Error budget for the DTI system

\begin{tabular}{l|c|c|c}
\hline Error source & Allowed & Effect on $\mathrm{V}(\times 1000)$ & Remark \\
\hline Wavefront error flats $(\lambda / \mathrm{N})$ & 20 & 120 & 9 per path \\
Wavefront error parabolic mirrors $(\lambda / \mathrm{N})$ & 5 & 277 & 3 per path \\
Off-axis errors $(\lambda / N)$ & 50 & 40 & \\
Unbalance $I_{1} / I_{2}$ & 0.7 & 16 & per two beams \\
OPD $(\mathrm{nm})$ & 20 & 80 & \\
Turbulence and vibrations $(\mathrm{nm})$ & 10 & 40 & per two beams \\
Polarization rotation $(\mathrm{rad})$ & 0.2 & 20 & \\
Lateral beam shift $(\mu \mathrm{m})$ & 3 & 48 & per two beams \\
Longitudinal beam shift $(\mathrm{mm})$ & 1 & 64 & \\
Beam rotation $(\mathrm{mrad})$ & 5 & 33 & \\
Pointing $(\mu \mathrm{rad})$ & 5 & 33 & \\
Magnification $(\Delta \mathrm{M} / \mathrm{M})$ & $1 \times 10^{-3}$ & 96 & \\
Stray light $\left(\mathrm{nW} / \mathrm{cm}^{2}\right)$ & 1 & 57 & \\
Drift lateral shift $(\mu \mathrm{m})$ & 2 & 32 & \\
Drift pointing $(\mu \mathrm{rad})$ & 20 & 33 & $\Delta\left(.8 \times 10^{-3}\right.$ \\
\hline Total visibility: & 0.314 & \multicolumn{2}{c}{}
\end{tabular}

middle optical path is taken as reference and the other two paths have to be controlled to be parallel to the middle one. The OPD can also only be adjusted for the two outer optical paths.

The created hardware is shown in Fig.5, at the end of this manuscript. 


\section{TRACKING}

One of the main difficulties in homothetic mapping is the real use of such a system. The telescopes that are combined will not have a constant arrangement as a function of the earth rotation angle. The baseline scales with a cosine factor due to the viewing direction. This means that when a star is followed during the night the arrangement of the mapping mirrors (in terms of the DTI system) will have to be changed continuously. Many schemes can be thought of on how to accomplish this rearranging of the mapping mirrors. The mapping requirement will again be in the order of several micrometers in lateral positioning. A stricter requirement is the optical path equality. During the whole observation time the OPD should be kept within the $10-50 \mathrm{~nm}$ range, depending on the required fringe visibility.

In DTI a novel approach to homothetic mapping can be tested (after implementing additional translational and rotational freedoms into the system). A patent is pending on this mapping approach. The idea behind this mapping approach is quite simple. Since the stars have to be viewed under changing directions as a function of time (due to earth rotation), the beams have to leave the mapping mirrors under the same angle. In this way the cosine factor is automatically taken care of and no difficult rearrangements of mirrors are necessary.

The first large flat mirror in the DTI, the Star Scanning Mirror (SSM) can be used to define the direction from which the simulated starlight is coming. The SSM will have to be translated and rotated, while the Aperture Mirrors will have to be rotated in order to direct the captured light correctly towards the delay lines. At the imaging side the mapping mirrors will have to be rotated accordingly and the Matching Scanning Mirror will have to match its movement with that of the SSM (hence its name). This operation describes a linear scan. For the simulation of a full 2D sky coverage the SSM should be able to reflect the light in all directions as would be expected in real sky observations. This would in turn require both translations and rotations of the SSM and the AM. These translations and rotations again have to be copied by the MM and MSM.

When applied to a real set of telescopes the viewing direction of the telescopes has to be copied by the mapping stage by rotation of the mirrors and translations of both the MSM (to reflect the light towards the imaging telescope) and the set of Mapping Mirrors. Since here the whole set of MMs is translated the configuration will remain equal to the configuration of the telescopes. The system can be designed such that both the MSM and the set of MMs need only a single translational degree of freedom and two rotational degrees of freedom.

For clarification of the concept, in Fig.3, an optical layout of a one dimensional scanning is shown. The observed object is assumed to describe a path that requires a rotation of the telescopes about the $y$-axis. In order to let the light enter the imaging telescope (paraboloid and hyperboloid surfaces), the large scanning mirror is translated (along the $x$-direction) and rotated (about $y$-axis), while the mapping mirrors are only rotated (about $y$-axis).

For a full sky coverage the scanning mirrors should also be rotatable about the $x$-axis with a scan along the $y$-direction. This should be accompanied by an extra rotational freedom of the MSM about the $x$-axis.

\section{EXPERIMENTS}

After the first alignment of the DTI setup the light will propagate from the light source (during the experiments this will be a Xe-lamp) all the way to the CCD camera. The first experiment will be to find the correct mapping condition. This will be done by scanning the delay lines and observing for which positions the star images show fringes. By observing the delay line settings as a function of the mapping mirrors layout the correct mapping layout can be found.

Once the proper mapper layout has been found it will be investigated how the fringe visibility depends in practice on the possible misalignments. During these experiments the OPD will be controlled actively by observing the fringes. The pointing of the three beams pertaining to the same star are not controlled. The direction should be stable enough owing to the mechanical stability. The found fringe visibility on misalignment will be compared to the theoretical dependencies.

The fibres are placed on a specially designed holder such that one fibre can be scanned while another fibre is stationary. We will measure the fringe visibility as a function of the fibre position (i.e. viewing direction of 

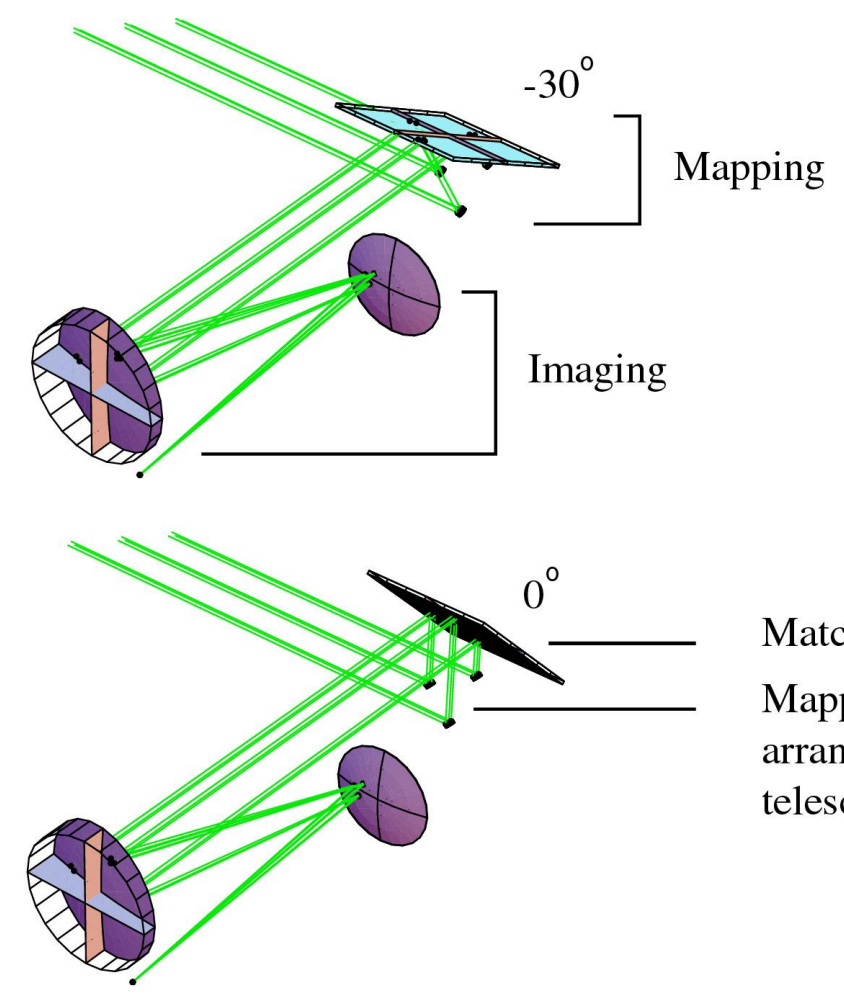

\section{Matching Scan Mirror \\ Mapping Mirrors in scaled arrangement of the telescopes}

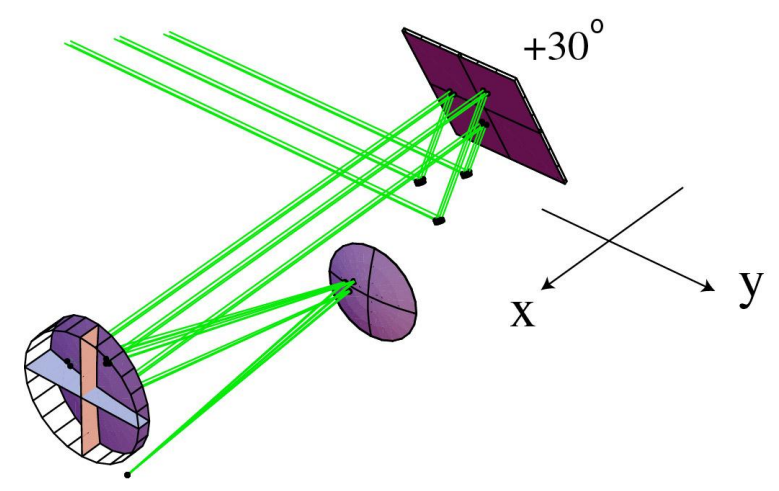

Figure 3. Optical layout of the mapping and imaging system of the DTI system. The layout is shown for three scanning angles, $-30^{\circ}, 0,30$, where the observed object is assumed to move directly overhead in a linear motion (rotation about the $y$-axis).

the DTI system, field of view). Once the change in visibility as a function of fibre position is known it will be measured how the DTI system has to be realigned (e.g. pointing adjustments) to enhance the visibility for all positions of the full field of view.

A further experiment will be to determine how poor the signal to noise ratio may be before phase retrieval from the fringe patterns is no longer possible. The $\mathrm{S} / \mathrm{N}$ ratio will be influenced by reducing the light intensity and by changing the spectral bandwidth of the light source (by inserting band filters in the beam path before the fibre). Figure 4 shows fringes as are simulated for two $10 \mathrm{~mm}$ width beams at a baseline of $100 \mathrm{~mm}$, being focused by a $5000 \mathrm{~mm}$ telescope (values as observed within DTI). For comparison the fringeless Airy pattern is also shown. It can be seen that for a narrow bandwidth the number of photons that can be used for the fringe phase calculations is far larger than for a wide bandwidth. 

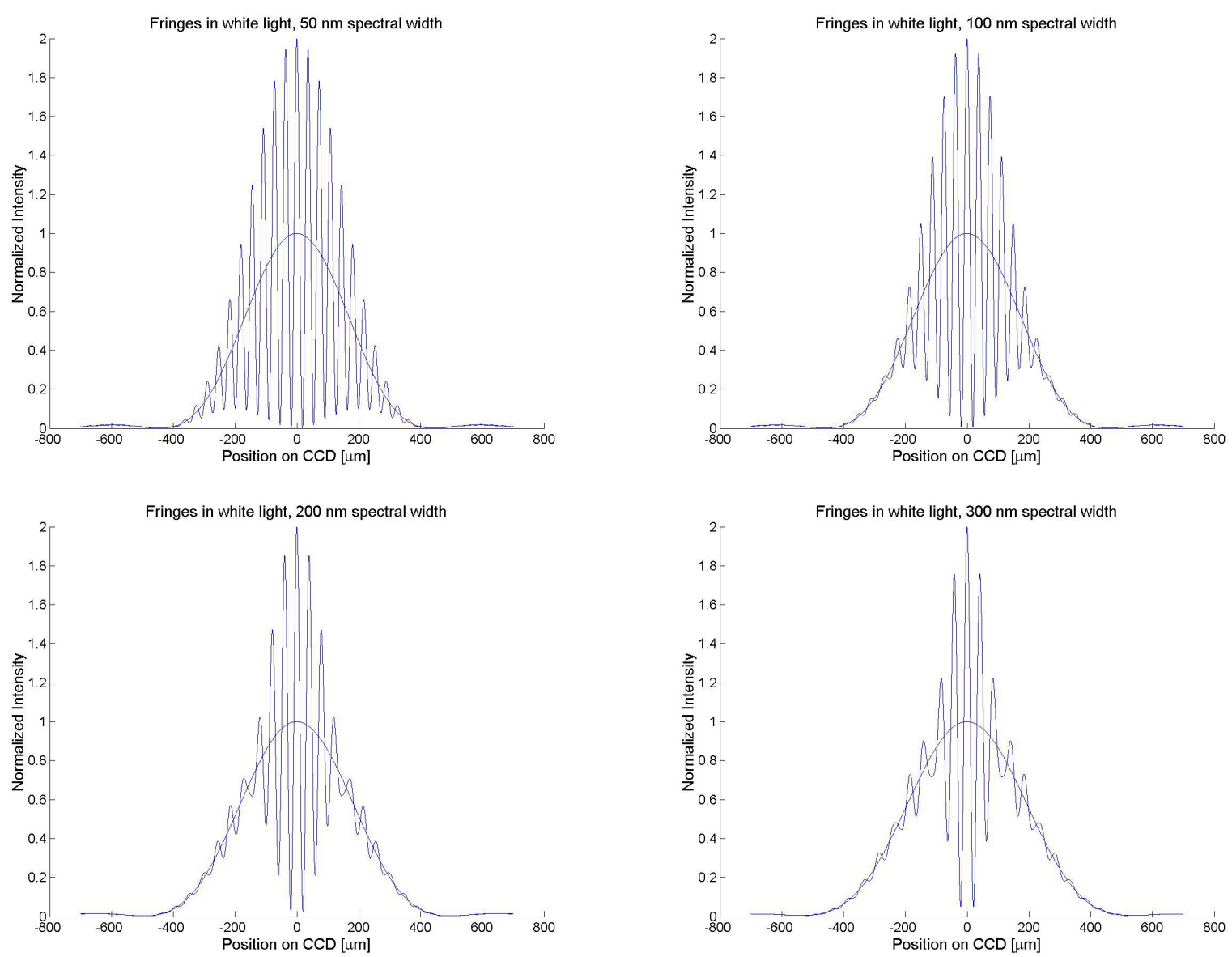

Figure 4. Fringe contrast for different spectral bandwidths.

Although the DTI system is designed to make use of the novel tracking system where only viewing angles are matched and where the configuration of the mirrors can be stationary, we decided it would be worthwhile to see whether a linear motion of one of the aperture mirrors can be followed by the corresponding mapping mirror. This experiment will give insight in the requirements for movements and whether they can be met by present day translation systems. The proposed operational scheme is to calibrate the mapping conditions for all positions of the scanned aperture mirror and then perform a blind tracking. This requires a strict reproducibility. The optical path differences will be monitored during the scanning operation and will be controlled actively. This means that the mapping reproducibility requirement will be in the order of micrometers and not in the nanometer range as would be needed without an active OPD control.

\section{CONCLUSIONS}

The main objectives of the Delft Testbed Interferometer have been presented together with the research goals. A complete error budget has been given and it has been shown that the fringe measuring accuracy can be better than one hundredth of the wavelength. The optical layout has been discussed and the novel tracking principle has been presented. Finally, the basic tracking experiment to be performed using the presented system is explained. 

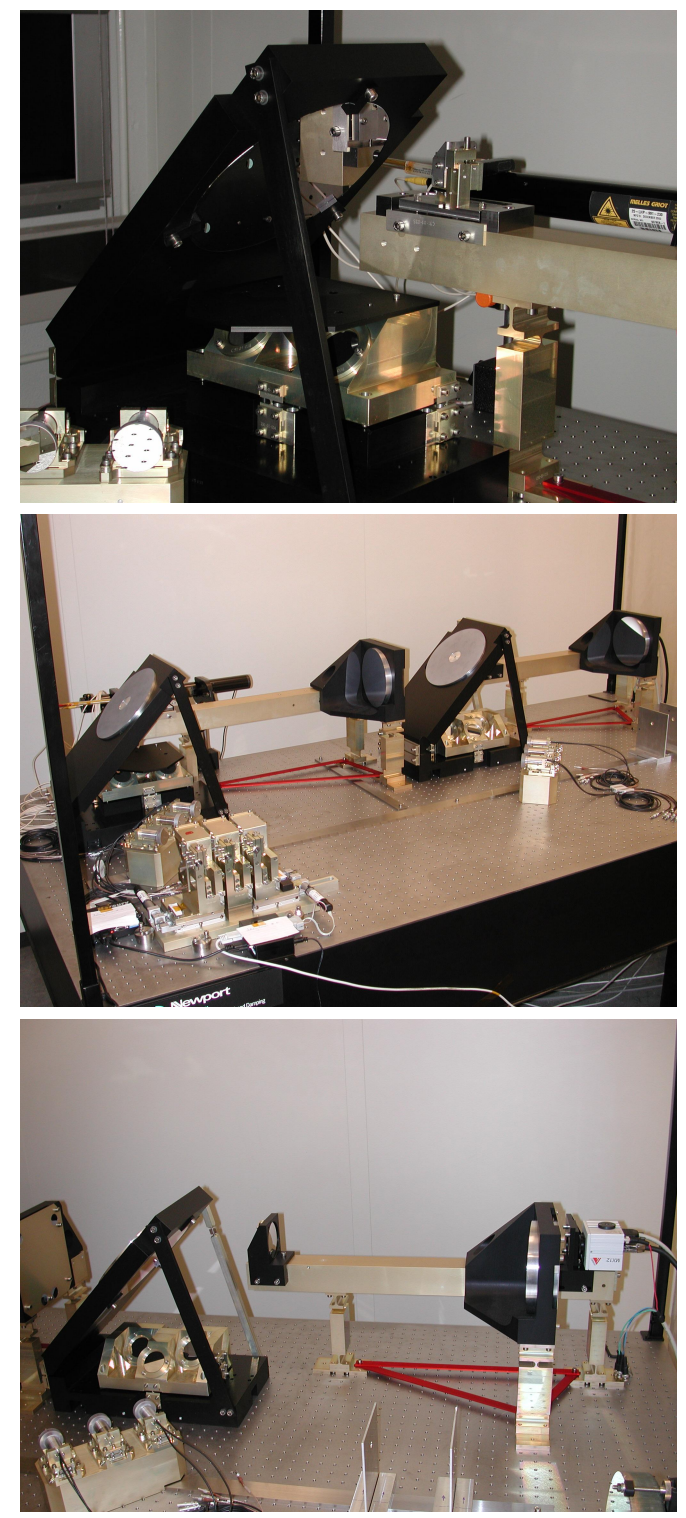

Figure 5. Photographs of the constructed hardware. From top to bottom the source are shown, a general overview, and the imaging telescope.

\section{REFERENCES}

1. Hedser van Brug, Teun van den Dool, Wim Gielesen, Peter Giesen, Bastiaan Oostdijck, and Luigi d'Arcio, "Delft Testbed Interferometer - layout design and research goals -", In Interferometry for Optical Astronomy II, edited by Wesley A. Traub, Proceedings of SPIE Vol 4838, pp.425 - 429 